\title{
Remarks on the Algebra of the 4-nodal Cubic Surface.
}

\author{
By W. SADDLER.
}

(Received 25th February 1929. Read 4th January 1929.)

The derivation of the 4-nodal Cubic Surface and the Quartic Surface, ${ }^{1}$ of which it is a particular case, are well known: certain new results of interest from the point of view of symmetry, and extension to $n$-fold space, are provided by the symbolic algebra. In particular a simple proof is given, in $\S 2$, of the theorem that a symmetry exists between the four vertices of the tetrahedron and the fifth point whose locus is the cubic surface, and this property can be extended to the case of $n+1$ points in $n$-fold space with one additional point.

$\$ 1$ Denote a quadric symbolically by the point equation $a_{x}{ }^{2}=a_{x}^{\prime}{ }^{2}=0$, or in plane coordinates by $u_{a}{ }^{2}=u_{a^{\prime}}{ }^{2}=\ldots 0$. The line equation to the quadric is $(A p)^{2}=\left(a a^{\prime} p\right)^{2}=0$ and two lines $q_{i j}, q_{i j}^{\prime}(i j=1,2,3,4, i \neq j)$ are conjugate lines to the quadric if

$$
(A q)\left(A q^{\prime}\right)=0, \ldots
$$

with analogous expressions for the tangential equation to the quadric.

The four-nodal cubic surface is the locus of a point $F$ such that the feet of the perpendiculars from $F$ to the faces of a tetrahedron $B C D E$ are coplanar. Or again-take the poles of the faces of the tetrahedron with respect to the quadric and join these poles to $F$ : such lines will meet the corresponding faces in four points which are coplanar if $F$ describes a quartic surface, which surface reduces to the cubic surface when the quadric is a conic in the plane at infinity. In $n$-fold space, $(n+1)$ points $B C D \ldots E$ are taken; and the poles of the faces with respect to a quadric: such points are joined to a further point $F$, and the lines meet the corresponding faces in points lying on a prime: $F$ will then describe a primal of order $(n+1)$, which reduces to a primal of order $n$-analogous to the 4-nodal cubic surface-when the quadric degenerates to a quadric of a prime.

\footnotetext{
'H. F. Baker, Principles of Geometry, 4, 26.
} 
Algebraically this means that the quadric surface, in plane coordinates, $u_{\alpha}^{2}=0$, is such that its discriminant vanishes, viz.,

$$
\left(a a^{\prime} a^{\prime \prime} a^{\prime \prime \prime}\right)^{2}=0
$$

and, taking $l_{x}=0$ to be the plane which contains the conic, then

$$
l_{a} \alpha_{i}=0 \quad(i=1,2,3,4),
$$

with corresponding expressions for $n$-space. A general quadric in $n$-space is a primal of order 2, while a prime is a primal of order unity.

Take the points $B C D E$ with coordinates $\beta, \gamma, \delta, \epsilon$, and equations,

where

$$
\begin{aligned}
& u_{\beta}=0, u_{\gamma}=0, u_{\delta}=0, u_{\varepsilon}=0, \\
& u_{\beta}=u_{1} \beta_{1}+u_{2} \beta_{2}+u_{3} \beta_{3}+u_{4} \beta_{4},
\end{aligned}
$$

and take any given plane $p_{x}=0$; then the equation of the quadric $S^{\prime}$ which passes through the curve of intersection of $S=0=a_{x}{ }^{2}$ and $p_{x}=0$ and also through the given four points is given by

$$
a_{x}^{2}(\beta \gamma \delta \epsilon)-p_{x}\left\{(x \gamma \delta \epsilon) \frac{a_{\beta}^{2}}{p_{\beta}}-(x \delta \epsilon \beta) \frac{a_{\gamma^{2}}}{p_{\gamma}}+(x \epsilon \beta \gamma) \frac{a_{\delta}^{2}}{p_{\delta}}-(x \beta \gamma \delta) \frac{a_{\epsilon}^{2}}{p_{\epsilon}}\right\}=0 ;
$$

as is easily verified. Calling this $a_{x}{ }^{2}(\beta \gamma \delta \epsilon)-p_{x} p_{x}^{\prime}=0$, the second factor $p_{x}^{\prime}$ thus gives the second plane of intersection of the two quadrics $S=0 \quad S^{\prime}=0$.

Reciprocally if $u_{a}^{2}=0$ be the quadric and four planes be given by $b_{x}=0, c_{x}=0, d_{x}=0, e_{x}=0$, and a point $F, u_{5}=0$, there is a second quadric envelope which touches the four planes and the cone, of vertex $F$, enveloping $u_{a}^{2}=0$. The vertex of the second enveloping cone, viz., $F^{\prime}$, to the two quadrics is given by $u_{\zeta}$, or,

$$
\text { (ucde) } \frac{b_{\alpha}^{2}}{b_{\zeta}}-(u d e b) \frac{c_{a}^{2}}{c_{\zeta}}+(u e b c) \frac{d_{\alpha}^{2}}{d_{\zeta}}-(u b c d) \frac{e_{a}^{2}}{c_{\zeta}}=0
$$

with analagous expressions for $n$-fold space. Then there will be $n+1$ such terms, and $n+1$ letters in each bracket factor.

Now take the poles of the four faces of $B C D E$ with respect to $u_{a}{ }^{2}=0$ : call these points $B^{\prime} C^{\prime} D^{\prime} E^{\prime}, B^{\prime}$ being the pole of $C D E$, and so on for the others. Such a point as $B^{\prime}$ has coordinates $(\gamma \delta \epsilon a) a_{i}$, or $b_{a} a_{i}$, where $b_{x}=0$ is the face $C D E$. The point, where the line joining $B^{\prime}$ to $F, u_{\zeta}=0$, meets the plane $b_{x}=0$, is given by,

$$
\zeta_{i} b_{a}^{2}-b_{\zeta} b_{\alpha} a_{i}
$$


Hence the four such points will be coplanar if the determinant, $\left(\zeta_{i} b_{\alpha}^{2}-b_{\zeta} b_{\alpha} a_{i}, \zeta_{i} c_{\alpha}{ }^{2}-c_{\zeta} c_{\alpha} \alpha_{i}, \zeta_{i} d_{\alpha}^{2}-d_{\zeta} d_{\alpha} \alpha_{i}, \zeta_{i} e_{\alpha}{ }^{2}-e_{\zeta} e_{\alpha} a_{i}\right)=0$. Or, on expanding,

$$
\begin{aligned}
\frac{1}{4 !}\left(a a^{\prime} a^{\prime \prime} a^{\prime \prime \prime}\right)^{2} & (b c d e) b_{\zeta} c_{\zeta} d_{\zeta} e_{\zeta} \\
& =\frac{1}{3 !} \sum_{b c d e}\left(\zeta a a^{\prime} a^{\prime \prime}\right)\left(c d e \mid a a^{\prime} a^{\prime \prime}\right) e_{\zeta} d_{\zeta} e_{\zeta} b_{a}{ }^{2} \ldots \ldots \ldots
\end{aligned}
$$

The right hand side involves 4 terms got by cyclical interchange of $b c d e$, but with positive and negative signs alternately.

Taking the point-form of the quadric $u_{a}^{2}=0$ as $a_{x}^{2}=0$, (5) can be written as

or

$$
\begin{array}{r}
a_{a}{ }^{2}(b c d e) b_{\zeta} c_{\zeta} d_{\zeta} c_{\zeta}=\sum_{\text {bcde }} a_{\zeta}(a c d e) c_{\zeta} d_{\zeta} e_{\zeta} b_{a}{ }^{2} . \\
\mathbf{1}=\sum_{\text {bede }} \frac{a_{\zeta}(a c d e) b_{\alpha}{ }^{2}}{b_{\zeta}(b c d e) a_{\alpha}{ }^{2}}, \ldots \ldots \ldots \ldots
\end{array}
$$

all the signs on the right being positive, the four terms being got by cyclical interchange of $b c d e$.

It is evident that $\zeta$ describes a quartic surface: in the case of $n$-fold space the relationship is exactly of the same form, viz.,

$$
\mathbf{l}=\sum_{b c d e \ldots . . .} \frac{a_{\zeta}(\text { acde } \ldots) b_{a}{ }^{2}}{b_{\zeta}(b c d e \ldots) a_{a}{ }^{2}} \ldots
$$

Expressing (7) in terms of the points $B C D E$, since by definition, $(b c d u)=u_{\epsilon}$, the equation can be written as

$$
1=\frac{\sum a_{\zeta} a_{\beta}(\gamma \delta \epsilon \alpha)^{2}}{a_{\alpha}^{2}(\beta \gamma \delta \epsilon((\gamma \delta \epsilon \zeta)}
$$

So the locus of $\zeta$ is

$$
1=\Sigma \frac{a_{x} a_{\beta}(\gamma \delta \epsilon \alpha)^{2}}{a_{a}^{2}(\beta \gamma \delta \epsilon)(\gamma \delta \epsilon x)}
$$

It is a straightforward application to show that, if condition (5) holds, then $F^{\prime} B^{\prime}, F^{\prime} B^{\prime}$ are conjugate lines to the quadric, where $F^{\prime}$ is the point whose equation is (4).

Let $F^{\prime}$ have coordinates $\zeta^{\prime}{ }_{i}$.

The lines $F B^{\prime}$ and $F^{\prime} B^{\prime}$ having coordinates

$$
(\zeta a)_{i j} b_{\alpha},\left(\zeta^{\prime} \alpha\right)_{i j} b_{\alpha},
$$


are conjugate with regard to $u_{\alpha}^{2}=0$ if

or

$$
\left(a a^{\prime} a^{\prime \prime} \zeta\right)\left(a a^{\prime} a^{\prime \prime \prime} \zeta^{\prime}\right) b_{a^{\prime \prime}} b_{a^{\prime \prime \prime}}=0,
$$

Substituting for $\zeta^{\prime}$ its coordinates from (4), and dividing by $b_{a}{ }^{2} \neq 0$, the condition (5) is obtained.

Similarly $F C^{\prime}, F^{\prime} C^{\prime}$ are conjugate lines, etc. It is clear that the proof holds for higher dimensions.

Let now $u_{\alpha}{ }^{2}=0$ reduce to a conic in the plane $l_{x}=0$, and hence

$$
\left(a a^{\prime} a^{\prime \prime} a^{\prime \prime \prime}\right)^{2}=0, l_{a} a_{i}=0 .
$$

Equation (5) becomes

$$
\sum_{b c d e}\left(a \alpha^{\prime} \alpha^{\prime \prime \zeta}\right)\left(a a^{\prime} a^{\prime \prime} \mid c d e\right) \frac{b_{\alpha}^{2}}{b_{\zeta}}=0
$$

Multiply both sides of the non-vanishing quantity $l_{\zeta}$ and notice that

$\left(\alpha a^{\prime} \alpha^{\prime \prime} \mid c d e\right) l_{\zeta}=\left(a \alpha^{\prime} \alpha^{\prime \prime} \zeta\right)(l c d e)+$ terms containing $l_{\alpha}$, which vanish. Thus a factor in each term is $\left(a a^{\prime} a^{\prime \prime} \zeta\right)^{2}$, and the remaining factor is.

or

$$
\frac{b_{\alpha}^{2}(l c d e)}{b_{\zeta}}-\frac{c_{\alpha}^{2}(l d e b)}{c_{\zeta}}+\frac{d_{\alpha}^{2}(l e b c)}{d_{\zeta}}-\frac{e_{\alpha}^{2}(l b c d)}{e_{\zeta}}=0
$$

$$
\frac{(\beta \gamma \delta \alpha)^{2} l_{\epsilon}}{(\beta \gamma \delta \zeta)}-\frac{(\gamma \delta \epsilon \alpha)^{2} l_{\beta}}{(\gamma \delta \epsilon \zeta)}+\frac{(\delta \epsilon \beta a)^{2} l_{\gamma}}{(\delta \epsilon \beta \zeta)}-\frac{(\epsilon \beta \gamma \alpha) l_{\delta}}{(\epsilon \beta \gamma \zeta)}=0 .
$$

thus the locus of $F$, or $\zeta$, is the known four-nodal cubic surface.

An analogous equation holds for the corresponding surface in $n$-space.

§2. It was first pointed out by W. Mantel' that each of the five points in (10), $B C D E F$, is in the same relation to the other four. This property holds similarly for $n$-space, as will now be shown from equation (10).

Now by the usual symbolic identity

$$
(\gamma \delta \epsilon a)^{2} l_{\beta}=(\gamma \delta \epsilon \alpha)\left\{(\gamma \delta \epsilon \beta) l_{\alpha}+(\gamma \delta \beta \alpha) l_{\epsilon}+(\gamma \beta \epsilon a) l_{\delta}+(\beta \delta \epsilon a) l_{\gamma}\right\}
$$

1 H. F. Baker, loco. cit. 
Now the first term on the right vanishes by $\S 1(3)$; take each of the other terms with the appropriate denominator of (10) from a corresponding one in (10): thus for example,

$$
\begin{aligned}
& \frac{(\gamma \delta \epsilon a)(\beta \gamma \delta a) l_{\epsilon}}{(\gamma \delta \epsilon \zeta)}-\frac{(\beta \gamma \delta \alpha)^{2} l_{\epsilon}}{(\beta \gamma \delta \zeta)} \\
& =\frac{(\beta \gamma \delta \alpha) l_{\epsilon}\{(\gamma \delta \epsilon \alpha)(\beta \gamma \delta \zeta)-(\beta \gamma \delta \alpha)(\gamma \delta \epsilon \zeta)\}}{(\gamma \delta \epsilon \zeta)(\beta \gamma \delta \zeta)} \\
& =\frac{(\beta \gamma \delta a) l_{\epsilon} .(\beta \gamma \delta \epsilon)(\gamma \delta \zeta \alpha)}{(\gamma \delta \epsilon \zeta)(\beta \gamma \delta \zeta)} .
\end{aligned}
$$

Each factor thus contains the non-vanishing $(\beta \gamma \delta \epsilon)$ and the total expression is

$$
\frac{(\beta \gamma \delta a)(\gamma \delta \zeta a) l_{\epsilon}}{(\beta \gamma \delta \zeta)}+\frac{(\epsilon \beta \gamma \alpha)(\epsilon \gamma \zeta \alpha) l_{\delta}}{(\epsilon \beta \gamma \zeta)}+\frac{(\delta \epsilon \beta a)(\delta \epsilon \zeta a) l_{\gamma}}{(\delta \epsilon \beta \zeta)}=0 .
$$

This expression is symmetrical in $\zeta$ and $\beta$ and thus the points $F$ and $B$ are interchangeable, which proves the result. For $n$-space there would be $n$ terms, each bracket factor containing $n+1$ symbols. 